\title{
Quantitative determination of peptides using matrix-assisted laser desorption/ionization time-of-flight mass spectrometry
}

Jesus A. Gutierrez, Jill A. Dorocke, Michael D. Knierman, Valentina Gelfanova, Richard E. Higgs, Nicholas Lewin Koh, and John E. Hale

Eli Lilly and Company, Indianapolis, IN, USA

BioTechniques 38:S13-S17 (June 2005)

A method is described for the quantitative determination of peptides using matrix-assisted laser desorption/ionization time-of-flight (MALDI-TOF) mass spectrometry. Known limitations imposed by crystal heterogeneity, peptide ionization differences, data handling, and protein quantification with MALDI-TOF mass spectrometry are addressed in this method with a "seed crystal" protocol for analyte-matrix formation, the use of internal protein standards, and a software package called maldi_quant. The seed crystal protocol, a new variation of the fast-evaporation method, minimizes crystal heterogeneity and allows for consistent collection of protein spectra. The software maldi_quant permits rapid and automated analysis of peak intensity data, normalization of peak intensities to internal standards, and peak intensity deconvolution and estimation for vicinal peaks. Using insulin proteins in a background of other unrelated peptides, this method shows an overall coefficient of variance of 4.4\%, and a quantitative working range of 0.58-37.5 ng bovine insulin per spot. Coupling of this methodology to powerful analytical procedures such as immunoprecipitation is likely to lead to the rapid and reliable quantification of biologically relevant proteins and their closely related variants.

\section{INTRODUCTION}

High-throughput genomics and proteomics technologies are identifying potential protein biomarkers at ever increasing rates. Orthogonal protein quantification techniques are needed to validate changes in protein levels indicated by these technologies. Typically antibody-based assays, such as the enzyme-linked immunosorbent assays (ELISA), radioimmunoassays (RIA), or quantitative Western blot analysis, are used to measure proteins in biological samples. Development of these assays is a time-consuming and resource-intensive effort. Moreover, results from these assays often provide quantitative observations for the total composition of the candidate proteins and do not discriminate among closely related members such as posttranslational protein variants. Development of simpler and quantitative methods could aid in the validation of protein biomarker candidates and help to triage biomarker candidates prior to undertaking development of these types of assays. Matrix-assisted laser desorption/ionization time-of-flight (MALDI-TOF) mass spectrometry has been used extensively for protein identification and characterization (1-3). However, it has been used less frequently for the quantification of proteins (4-11). Numerous published references describe the limitations of MALDI-TOF for quantitative purposes $(4,7,12)$. These include: (i) analyte-matrix heterogeneity, which contributes to poor reproducibility of signals; (ii) differential ionization efficiency of proteins; (iii) variability in the surface of the MALDI-TOF target; (iv) limited dynamic range due to saturation of the mass spectrometer detector; and $(v)$ difficulty extracting and processing easily, reliably, and with high-throughput the quantitative components, such as peak area or intensity, from the spectra collected. The published reports for quantitative MALDI-TOF begin to address the problem of analytematrix heterogeneity, limitations on the dynamic range due to saturation of the mass spectrometer detector, and to some degree the differential ionization efficiency of proteins. To address analytematrix heterogeneity, two basic approaches are described: (i) the use of a diversity of matrices to complex the analyte, including binary matrix complexes such as fucose and ferulic acid and (ii) a fast evaporation method to layer a thin coating of matrix on the target surface for analyte crystallization (12-15). Significant improvements on the previously described crystal-to-crystal variability are described with both of these approaches, yet limitations still are apparent. First, the appropriate matrix or a combination of matrices June 2005 must be identified to reach the optimal ratio and composition for reliable quantitative MALDI-TOF mass spectrometry analysis. Second, the target surfaces described for these approaches often encompass small working areas $(<10 \mathrm{~mm})$ with capacity to carry few spots for MALDI-TOF analysis. Nordhoff and colleagues (12) have described a method amenable for 96- or 384-well target plates, in which a hand-held spreader is used to spread the matrix solution over the plates. An apparent limitation for this method is the potential for uneven distribution of matrix material over the target plate as a result of inconsistent hand motion over the plate. To address the problems associated with variable protein ionization, peak intensity normalization approaches have been described, however, the normalization protocols often use unrelated proteins as the normalization standards making the assumption that the chosen standards behave consistently in relation to the peptide of interest $(4,8,16)$.

Other methods have been developed in the last few years to quantify protein levels using mass spectrometry (17-19). These methods have primarily aimed at electrospray ionization techniques. A number of these methods have used stable isotope labeling of proteins and peptides to quantify complex proteins mixtures (17-19). A limitation with these approaches is the significant amount of sample preparation often required prior to analysis and the length of time needed to fractionate and assay each sample. Of interest, these procedures do describe the use of stable isotopes, such as ${ }^{13} \mathrm{C}$ and $15 \mathrm{~N}$ containing amino acids, to label peptides or proteins. These labeled proteins, which are chemically identical to the proteins of interest, are easily detected due to their mass differences, can be used to account for sample processing efficiencies, and provide an approach to quantify proteins of interest. An example for the use of labeled proteins for quantitative purposes is the inclusion of isotopomers for the protein(s) of interest with efficient immunoprecipitation methods to measure proteins in biological samples using MALDI-TOF mass spectrometry detection (20).

In this manuscript, we address each of the described limitations for quantitative MALDI-TOF as follows: (i) the problem of analytematrix heterogeneity or limited surface area is overcome using a rapid matrix spreading technique that provides an even distribution of "seed crystals" for the analyte-matrix in large MALDI-TOF target surfaces, such as 100-well plates; (ii) we address the concern of ionization variation among proteins by using closely related proteins as normalization standards; (iii) we minimize saturation of the mass 
spectrometer detector by utilizing settings in the mass spectrometer where saturation is not likely to occur; and (iv) we utilize a software program capable of rapidly and efficiently identifying candidate peaks, determining the baseline for each peak, calculating the area for each peak, and defining the normalization ratios for the proteins of interest. In addition, we subject data collected under this new method to a stringent statistical analysis to specifically estimate the sources of variation and the quantitative ranges for the assay.
Table 1. Comparison of Coefficient of Variation for the Two MALDI Plate Surface Preparation Methods

\begin{tabular}{|cccc|}
\hline Metric & Analyte & $\begin{array}{c}\text { Seed Crystal CV } \\
(\mathbf{\%})\end{array}$ & $\begin{array}{c}\text { Bare Surface CV } \\
(\mathbf{\%})\end{array}$ \\
\hline Spectrum peak areas & Bovine insulin & 34 & 119 \\
Spectrum peak areas & Human insulin & 34 & 97 \\
Spot-level average peak area & Bovine insulin & 17 & 36 \\
Spot-level average peak area & Human insulin & 18 & 29 \\
Ratio of spot-level average & Bovine/Human & 1.2 & 10 \\
Spectrum peak area metrics correspond to the coefficients of variation (CVs) estimated among the \\
respective spectrum peak intensity averages, and the spot level metrics represent the CV for the peak area \\
intensities for the spot level averages. MALDI, matrix-assisted laser desorption/ionization.
\end{tabular}

\section{MATERIALS AND METHODS}

$\alpha$-Cyano-4-hydroxycinnamic acid, methanol, bovine insulin, human insulin, glucagon-like peptide-1 (GLP-1; 1-36), GLP-1 (7-36), adrenocorticotropic hormone (ACTH; 18-39), and bovine insulin $\beta$-chain peptides were purchased from Sigma (St. Louis, MO, USA). Rat parathyroid hormone (PTH; 1-34) was purchased from Peninsula Laboratories (Belmont, CA, USA). Fatty acid amide hydrolase (FAAH peptide; H-VGYYETDNYTMPSPAMR-OH) was synthesized at Lilly Research Laboratories (Eli Lilly and Company, Indianapolis, IN, USA). Trifluoroacetic acid (TFA) was obtained from Pierce Biotechnology (Rockford, IL, USA). Acetonitrile was from Burdick and Jackson (Muskegon, MI, USA).

\section{Sample Preparation}

Peptide solutions and dilutions were made in $0.1 \%$ TFA. For spotting on the MALDI-TOF stainless steel target plate, peptide dilutions were mixed at a ratio of 1 part peptide mixture at the desired concentration to 3 parts of a saturated solution of $\alpha$-cyano4-hydroxycinnamic acid in 50\% acetonitrile and $0.05 \%$ TFA. One microliter volume for each sample solution was spotted directly onto the target plate, and for each spot analyzed, 12 spectra were

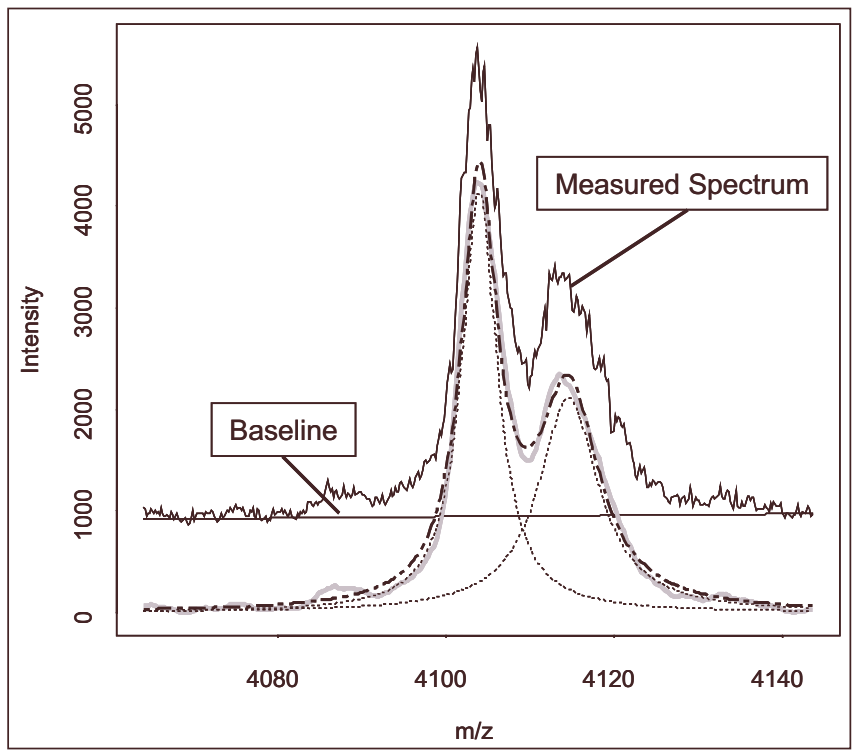

Figure 1. Rat parathyroid hormone (PTH) and glucagon-like peptide-1 (GLP-1; 1-36) peak fitting results. Measured spectrum, local linear baseline, smoothed and baseline corrected spectrum (gray), rat PTH and GLP-1 1-36 deconvolved components (dotted), and sum of fitted components (dashed). $\mathrm{m} / \mathrm{z}$, mass-to-charge ratio. collected using the automated feature of the MALDI-TOF mass spectrometer.

To determine the variance components and the quantitative range for the assay, the following three experiments were implemented: (i) a seed crystal comparison study; (ii) a variance components study; and (iii) a spiked recovery study. To implement the seed crystal comparison study, human and bovine insulin proteins were spotted at $1 \mathrm{ng}$ each per spot on 5 distinct spots of two plates. Both plates were cleaned as described below. However, one plate was coated with seed crystals while the second plate was used without seed crystals. The variance component study consisted of a 3-day, 4 plates/day, 5 spots/plate, and 12 readings/spot on plates coated with seed crystals.

FAAH peptide was used at $200 \mathrm{pg} / \mathrm{spot}$, and GLP-1 1-36, GLP-1 $7-36$, and rat PTH were used at $500 \mathrm{pg} / \mathrm{spot}$. ACTH was used at $50 \mathrm{pg} / \mathrm{spot}$, and bovine insulin $\beta$-chain was used at $750 \mathrm{pg} / \mathrm{spot}$. Human and bovine insulin proteins were used at $1 \mathrm{ng} / \mathrm{spot}$. The spiked recovery experiment was composed of data collected on 3 plates, 3 standard curves/plate composed of 9 points/curve. In addition, a quadruplicate set of validation samples at eight different levels relative to the standard curve was included in each plate. For the standards, all proteins were spotted as described for the variance components study, except for human insulin, which was used at $1.56 \mathrm{ng} / \mathrm{spot}$. In addition, the bovine insulin protein was added at the following levels: 50, 25, 12.5, 6.25, 3.125, 1.56, 0.78, 0.39 , and $0.2 \mathrm{ng} / \mathrm{spot}$. The validation samples were at $37.5,18.75$, $9.38,4.69,2.34,1.17,0.58$, and $0.29 \mathrm{ng} / \mathrm{spot}$.

\section{Mass Spectrometry}

A Voyager DE-PRO MALDI-TOF mass spectrometer (PerSeptive Biosystems, Framingham, MA, USA) was used for analysis in the linear delayed extraction mode. The laser was operated at a fixed fluence just above the threshold value. The internal digitizer was set to the following parameters: bin size was $1 \mathrm{~ns}$, full-scale set to $500 \mathrm{mV}$, full bandwidth mode, and a vertical offset of $1.2 \%$ full-scale. Spectra were acquired with the following parameters: low mass gate set to $1000 \mathrm{Da}$, mass range set to $1000-10,000 \mathrm{Da}$, accelerating voltage set to $20,000 \mathrm{~V}$, grid voltage set to $95 \%$ of accelerating voltage, guide wire set to $0.2 \%$ of the accelerating voltage, and the delayed extraction time was set to $300 \mathrm{~ns}$. Twelve independent spectra were automatically collected for each spot by a random, center-biased pattern.

\section{Preparation of Matrix Surfaces}

The 100-well stainless steel MALDI-TOF target plates (PerSeptive Biosystems) were washed and scrubbed gently in the following solutions and order: (i) 100\% ethanol scrub; (ii) deionized 
water rinse; (iii) Contrad-100 detergent scrub (Decon Laboratories, King of Prussia, PA, USA); (iv) deionized water rinse; (v) 100\% ethanol rinse; and (vi) 100\% methanol rinse. Subsequently, the plates were dried at room temperature. To layer the thin coating of matrix solution or seed crystals on the plates, clean target plates were placed on a laboratory magnetic $6 \times 6$ stirrer (Cole-Parmer, Chicago, IL, USA), modified to safely expose the magnet and stably hold the target plate. The plates were spun at a setting of 4 on the spinner (medium speed), and $150 \mu \mathrm{L}$ methanol-matrix solution were added directly to the spinning plate. The plate was allowed to continue spinning for an additional $15-30$ s to ensure even distribution and complete evaporation of the methanol-matrix solution on the plate surface. To generate the methanol-matrix solution, recrystallized $\alpha$-cyano-4-hydroxycinnamic acid matrix was dissolved at its saturation point in $100 \%$ methanol at room temperature. The saturated matrix solution was further diluted to $3.75 \%$ final concentration in methanol and applied to the plate. Analyte mixtures in saturated matrix, as described above, were spotted on the plate and allowed to dry at room temperature prior to analysis.

\section{Data Acquisition and Processing}

Software for data analyses post acquisition from the MALDI-TOF mass spectrometer was written in-house using the $R$ statistical computing environment (21). The inputs to this internally developed R procedure, maldi_quant, include a list of peptide massto-charge ratio $(\mathrm{m} / \mathrm{z})$ values to quantify and a spectrum or a directory of spectra. Normal processing of the mass spectra included mass calibration, smoothing of the spectrum using a super smoother (22), local linear baseline subtraction, and nonlinear regression peak fitting with a Lorentzian peak shape for quantification. Nonlinear regression was carried out using Gauss-Newton optimization as implemented in the $\mathrm{R}$ function $n / s$ (23). Overlapping peaks (rat PTH and GLP-1 1-36) were fit using a mixture of Lorentzian peaks in order to deconvolve the peak area from each component (Figure 1). Initial estimates of the Lorentzian peak parameters, location, scale, and area were derived from the spectrum in a region around a local maximum.

\section{RESULTS}

\section{Effect of Seed Crystals on Plate Surface and Spot Appearance}

Coating of seed crystals using the spinning method described here created a thin translucent layer of matrix on the entire MALDITOF target plate. The impact of this thin coating of seed crystal was more apparent on the shape and consistency of the analytecrystal complexes once dried on the plate. Layering of seed crystals caused the analyte-matrix complexes to form rapidly and evenly over the entire spot area (data not shown). Upon analysis in the MALDI-TOF mass spectrometer, signals of excellent quality and consistency were observed throughout the entire spot independent of spot location. In contrast, analyte-matrix crystals generated on plates lacking the seed crystals, as expected, were slow to form, had a heterogeneous boulder-like appearance, and were randomly distributed over the spot. In addition, "sweet spot" areas or crystal regions with particularly strong signals could be readily identified among the analyte-matrix complexes.

In order to quantify the impact of seed crystals on the quality of the signals observed, a small seed crystal comparison study was implemented. The study consisted of two plates containing 5 spots with $1 \mathrm{ng} / \mathrm{spot}$ each for bovine and human insulin proteins. One plate was layered with the seed crystals while the second had the bare surface for analyte-matrix formation. Peak areas from individual spectra, spot-level average peak areas, and
Table 2. Variance Components Estimates for the Seed Crystal Surface Preparation Method

\begin{tabular}{|cc|}
\hline Variance Component & $\begin{array}{c}\text { Proportion of } \\
\text { Total Variance } \\
\text { (\%) }\end{array}$ \\
\hline Day & 0 \\
Plate[Day] & 34 \\
Spot[Plate*Day] & 23 \\
Residual (Spectrum-to-Spectrum) & 43 \\
Overall coefficient of variation (CV) for the spot level average peak area \\
ratios was 4.4\%. The CV for both bovine and human insulin peak areas \\
was 35\%. Relative contributions of each step to total variance was the \\
same for both bovine and human insulin.
\end{tabular}

spot-level ratio of average peak areas for each protein were estimated using the maldi_quant software. At both the individual spectrum and spot-level quantification, the seed crystal surface preparation demonstrated considerably better precision (Table 1). The spot level average peak area ratio coefficients of variance were $1.2 \%$ and $10 \%$ for the seed crystal and bare surface control treatments, respectively. In order to gain a better understanding of the variance components for the seed crystal treatment, a multiday, multiplate, and multispot experiment was performed. For this study, a mixture composed of the eight proteins described in the Materials and Methods section at levels calculated to give reasonable signals were spotted on 5 spots, repeated on 4 plates, and run on 3 different days. The peak areas and intensity ratios for the bovine and human insulin proteins, in the background of other unrelated proteins, were calculated with the software package maldi_quant and analyzed to estimate the total variance, the amount of variance imparted by each component of the experiment, and the overall coefficient of variation. A random effects model with the terms day, plate [day], spot [day*plate] and residual (spectrum) was fit using the JMP Statistical Discovery Software ${ }^{\circledR}$ package version 5.1.1 (SAS Institute, Cary, NC, USA), using restricted maximum likelihood. The results from this experiment indicated that the contribution to total peak area variability is approximately evenly split between plate, spot, and spectrum (Table 2). A random effects model fit with the ratio of spot-level averages for bovine and human insulin resulted in an estimated overall coefficient of variation of $4.4 \%$. To estimate a quantification working range for a prototypical MALDI-TOF based assay, we implemented a spiked recovery experiment composed of a 3-plate, 9-point standard curve (run in triplicate), and 8 validation samples at concentrations between those used in the standard curve (run in quadruplicate) per plate. For this experiment, the levels for the background proteins ranged from $50 \mathrm{pg}$ to $1.56 \mathrm{ng} /$ spot, as previously described, and the concentration of the bovine insulin protein varied (see Figure 2). We chose the concentrations of bovine insulin for this study, based on our experience with the protein, to ensure that we spanned a range in concentration from just below the limit of detection to near saturation. Figure $2 \mathrm{~A}$ shows a representative dose-response relationship for the concentration of bovine insulin protein to the peak area ratio between the bovine and human insulin protein peaks. Calibration curves were fit using a four-parameter logistic model with the nonlinear regression feature in the JMP statistical software package. Variance components were estimated for the between and within plate effects, and the total error was decomposed into bias and precision components (Figure 2B). Results from this analysis showed a 64-fold range in which the total error coefficient of variance was less than $30 \%$ (0.58-37.5 ng/spot). 

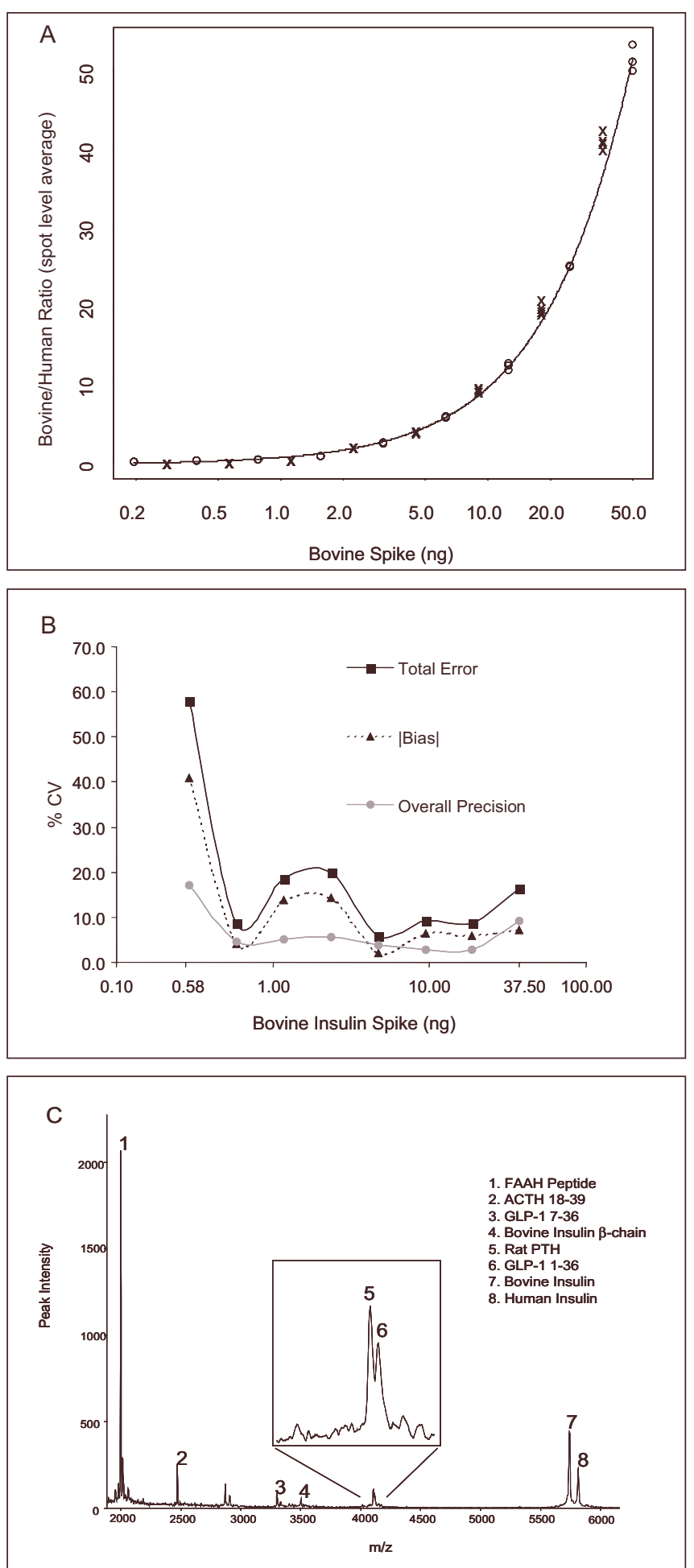

Figure 2. Results for spiked recovery experiment. (A) Plate no. 1 instrument response for bovine insulin titration. Calibration dilutions denoted by open circles $(n=3$ replicates/dilution) and validation dilutions denoted by Xs $(n=4$ replicates/dilution). Four parameter logistic fit based on calibration dilutions shown. (B) Bias, precision, and total error estimated from the between and within plate spiked recovery runs. Defining an assay working range by total error less than $30 \%$ yields a working range of approximately 64 -fold $(0.58-37.5 \mathrm{ng} / \mathrm{spot})$. (C) Representative spectrum used to generate working curve for spike-recovery experiment. For this particular spectrum, the bovine insulin protein was at $3.12 \mathrm{ng} / \mathrm{spot}$, while the rest of the protein amounts were as described under the Materials and Methods section. Peaks 1-8 represent the eight proteins used in these studies, and the inset reveals a close view for the peaks corresponding to the rat parathyroid hormone $(\mathrm{PTH})$ and glucagon-like peptide1 (GLP-1). CV, coefficient of variance; $\mathrm{m} / \mathrm{z}$, mass-to-charge ratio; FAAH, fatty acid amide hydrolase; ACTH, adrenocorticotropic hormone.

\section{DISCUSSION}

The principal factors limiting the capability of MALDI-TOF mass spectrometry as a quantitative tool include analyte-matrix heterogeneity, differential ionization efficiency of proteins, saturation of the mass spectrometer detector, and difficulty processing the quantitative components from the large amounts of data generated by mass spectrometry. In this work, we describe a method that minimizes the analyte-matrix heterogeneity by precoating the MALDI-TOF target plates with a thin layer of $\alpha$-cyano-4-hydroxycinnamic acid matrix seed crystals. This target plate treatment causes the protein-matrix crystals to form uniformly over the entire surface of the target spot ensuring a consistent generation of spectra independent of crystal-to-crystal effects. It is thought that the seed crystals function as focal points for the rapid and simultaneous growth of the analyte-matrix complexes on the surface of the target plate. This rapid rate of crystal formation may minimize kinetic or hydrophobic driven preferential crystallization effects of proteins from a complex protein mixture producing a lawn of homogeneous crystals (13). Gusev and coworkers (13) have shown improvements in signal reproducibility with other matrices such as sinapinic acid, ferulic acid, caffeic acid, gentisic acid, and 3-hydroxypicolinic acid, including $\alpha$-cyano-4-hydroxycinnamic acid. Therefore, it would be important to determine if the improvements observed with the seed crystal procedure reported here could also be achieved with commonly used matrices. The most obvious benefit of this procedure is not only the ability to collect consistent readings anywhere in the spot of interest, but also the rate in which these data can be collected, since this procedure does not necessitate the search for sweet spots on the analyte/matrix crystals. We addressed the limitation caused by the differential ionization efficiency of proteins with the inclusion of internal protein standards (17-19). For this work, we use a collection of biological proteins as the background source of protein ionization. However for quantification purposes, we use human insulin as the internal reference standard against bovine insulin, and we describe the peak area ratios for quantitative purposes. Ideally, the use of isotopomers, standard proteins labeled with ${ }^{2 \mathrm{H}},{ }^{13} \mathrm{C},{ }^{15} \mathrm{~N}$, or other isotopes, is recommended for quantification purposes, since these isotope-labeled proteins are easily detected in the MALDI-TOF mass spectrometer due to their expected mass differences and behave identically to the protein of interest. In order to prevent saturation of the mass spectrometer detector, we use protein concentrations and settings well below the known saturation points. Finally, the problem of data extraction and manipulation from large collections of spectra was addressed in this work by the use of the software program maldi quant. This program identified the peaks of interest, determined the baseline for each peak, estimated the peak areas, and calculated the peak intensity ratios for bovine to human insulin. Fitting a parametric peak shape to the data enables us to quantify nonbaseline resolved peaks in the spectrum, an important feature when related proteins or internal standards may be close in mass to the analyte of interest.

Statistical analysis comparing and contrasting the impact of the seed crystals on the bovine insulin peak intensity ratio decreased the overall coefficient of variance from $10 \%$ to $1.2 \%$. In addition, analysis for the proportion of variance contributed by day, plate, spot, and the residual effects showed that the individual components of variance are approximately evenly distributed between plate, spot, and residual. Statistical analysis of the seed crystal method also demonstrated the quantitative working range for the assay, at least for bovine insulin. This assay showed a working range (as defined by total error coefficient of variation less than $30 \%$ ) between 0.58 and $37.5 \mathrm{ng} / \mathrm{spot}$ as shown by the total error profile (Figure 2B).

There are at least two practical applications for this technology. In the discovery setting, coupling of quantitative MALDI-TOF mass spectrometry to sensitive and accurate immunoprecipitation 
procedures can be used not only for the quantification of bioanalytes, including proteins with high degrees of accuracy and sensitivity, but also in the identification and quantification of closely related proteins. Examples of this argument include the use of quantitative MALDI-TOF mass spectrometry to study posttranslational or splice variants for proteins of interest. In addition, data generated from quantitative MALDI-TOF mass spectrometry can discern the relative contribution that related bioanalytes give to the overall signal observed. This is clearly an improvement over quantitative assays, such as ELISA or RIA, where measurements observed correspond to the sum of all the components involved. In a clinical setting, this methodology may have direct applications because of its consistency, high capacity, quantitative potential, and amenability to automation. In addition, quantitative MALDI-TOF mass spectrometry coupled to immunoprecipitation procedures has the potential for simultaneous quantitative analysis of multiple bioanalytes.

In summary, we describe a seed crystal method that, when used in conjunction with internal standards, appropriate instrument settings, and the software program maldi_quant, addresses the known limitations for quantitative MALDI-TOF mass spectrometry. The sensitivity, precision, and accuracy parameters for this assay are determined via a comprehensive statistical analysis and demonstrate the potential new utilities of quantitative MALDI-TOF mass spectrometry for bioanalytical purposes.

\section{ACKNOWLEDGMENTS}

We wish to thank Drs. Jeffrey S. Patrick and Viswanath Devanarayan for helpful discussion and input on the topics of mass spectrometry and assay validation and characterization.

\section{COMPETING INTERESTS STATEMENT}

The authors declare no competing interests.

\section{REFERENCES}

1. Mann, M. and G. Talbo. 1996. Developments in matrix-assisted laser desorption/ ionization peptide mass spectrometry. Curr. Opin. Biotechnol. 7:11-19.

2. Andersen, J.S. and M. Mann. 2000. Functional genomics by mass spectrometry. FEBS Lett. 480:25-31.

3. Tang, K., S.L. Allman, R.B. Jones, and C.H. Chen. 1993. Quantitative analysis of biopolymers by matrix-assisted laser desorption. Anal. Chem. 65:2164-2166.

4. Bucknall, M., K.Y.C. Fung, and M.W. Duncan. 2002. Practical quantitative biomedical applications of MALDI-TOF mass spectrometry. J. Am. Soc. Mass Spectrom. 13:1015-1027.

5. Bilati, U., C. Pasquarello, G.L. Corthals, D.F. Hochstrasser, E. Allemann, and E. Doelker. 2005. Matrix-assisted laser desorption/ionization time-of-flight mass spectrometry for quantitation and molecular stability assessment of insulin entrapped within PLGA nanoparticles. J. Pharm. Sci. 94:688-694.

6. Gobom, J., K.O. Kraeuter, R. Persson, H. Steen, P. Roepstorff, and R. Ekman. 2000. Detection and quantification of neurotensin in human brain tissue by matrixassisted laser desorption/ionization time-of-flight mass spectrometry. Anal. Chem. 72:3320-3326.

7. Mirgorodskaya, O.A., Y.P. Kozmin, M.I. Titov, R. Korner, C.P. Sonksen, and P. Roepstorff. 2000. Quantitation of peptides and proteins by matrix-assisted laser desorption/ionization mass spectrometry using ${ }^{18} 0$-labeled internal standards. Rapid Comm. Mass Spectrom. 14:1226-1232.

8. Babu, C.V.S., J. Lee, D.S. Lho, and Y.S. Yoo. 2004. Analysis of substance P in rat brain by means of immunoaffinity capture and matrix-assisted laser desorption/ ionization time-of-flight mass-spectrometry. J. Chromatogr. B 807:307-313.

9. Nedelkov, D., R.W. Nelson, U.A. Kiernan, E.E. Niederkofler, and K.A. Tubbs. 2003. Detection of bound and free IGF-1 and IGF-2 in human plasma via biomolecular interaction analysis mass spectrometry. FEBS Lett. 536:130-134.

10. Helmke, S.M., C. Yen, K.J. Cios, K. Nunley, M.R. Bristow, M.W. Duncan, and M.B. Perryman. 2004. Simultaneous quantification of human cardiac $\alpha$ - and $\beta$-myosin heavy chain proteins by MALDI-TOF mass spectrometry. Anal. Chem. 76:1683-1689.

11. Biroccio, A., A. Urbani, R. Massoud, C. di llio, P. Sacchetta, S. Bernardini, C. Cortese, and G. Federici. 2005. A quantitative method for the analysis of glycated and glutathionylated hemoglobin by matrix-assisted laser desorption ionization-time of flight mass spectrometry. Anal. Biochem. 336:279-288.
12. Nordhoff, E., M. Schurenberg, G. Thiele, C. Lubbert, K. Kloeppel, D. Theiss, H. Lehrac, and J. Gobom. 2003. Sample preparation protocols for MALDI-MS of peptides and oligonucleotides using prestructured sample supports. Int. J. Mass Spectrom. 226:163-180.

13. Gusev, A.I., W.R. Wilkinson, A. Proctor, and D.M. Hercules. 1995. Improvement of signal reproducibility and matrix comatrix effects in MALDI analysis. Anal. Chem. 67:1034-1041.

14. Vorm, O., P. Roepstorff, and M. Mann. 1994. Improved resolution and very high sensitivity in MALDI TOF of matrix surfaces made by fast evaporation. Anal. Chem. 66:3281-3287.

15. Cohen, S.L. and B.T. Chait. 1996. Influence of Matrix solution conditions on the MALDI-MS analysis of peptides and proteins. Anal. Chem. 68:31-37.

16. Nelson, R.W., M.A. McLean, and T.W. Hutchens. 1994. Quantitative determination of proteins by matrix-assisted laser desorption/ionization time-of-flight mass spectrometry. Anal. Chem. 66:1408-1415.

17. Lill, J. 2003. Proteomic tools for quantitation by mass spectrometry. Mass Spectrom. Rev. 22:182-194

18. Sechi, S. and Y. Oda. 2003. Quantitative proteomics using mass spectrometry Curr. Opin. Chem. Biol. 7:70-77.

19. Tao, W.A. and R. Aebersold. 2003. Advances in quantitative proteomics via stable isotope tagging and mass spectrometry. Curr. Opin. Chem. Biol. 14:110-118.

20. Hess, J.L., L. Blazer, T. Romer, L. Faber, R.M. Buller, and M.D.P. Boyle. 2005. Immunoproteomics. J. Chromatogr. B. 815:65-75.

21.R Development Core Team. 2004. R: A language and environment for statistical computing. R Foundation for Statistical Computing, www.R-project.org.

22.Friedman, J.H. 1984. A variable span scatterplot smoother. Laboratory for Computational Statistics, Stanford University, Technical Report no. 5.

23. Bates, D.M. and D.G. Watts. 1988. Nonlinear Regression Analysis and Its Applications. Wiley \& Sons, New York.

Address correspondence to:

Jesus A. Gutierrez

Integrative Biology, Applied Biochemistry/Proteomics

Bldg. 220; GL 54

Eli Lilly and Company

2001 W. Main Street; P.O. Box 708

Greenfield, IN 46140, USA

e-mail: Gutierrez_jesus_a@lilly.com 